\title{
Development and Verification of a CMOS Phototransistor Noise Model
}

\author{
Plamen Kostov, Wolfgang Gaberl, Michael Hofbauer and Horst Zimmermann \\ Institute of Electrodynamics, Microwave and Circuit Engineering \\ Vienna University of Technology \\ Vienna, Austria \\ [plamen.kostov | wolfgang.gaberl | michael.hofbauer | horst.zimmermann]@tuwien.ac.at
}

\begin{abstract}
In this paper a noise model for phototransistors is presented. Noise measurements and Gummel measurements on four different phototransistors were performed to verify the noise model. In addition, the output noise current density was modeled and compared with the measurements. A maximum difference of less than $12 \%$ is noticed.
\end{abstract}

Keywords-phototransistor; noise; noise model; shot noise; gummel; current gain; pnp; pin;

\section{INTRODUCTION}

Phototransistors (PTs) are promising devices for detection of light. Compared to $\mathrm{p}(\mathrm{i}) \mathrm{n}$ photodiodes they have an inherent gain. Together with a suitable readout circuitry they offer possibilities for different applications, e.g. shear sensors, image sensors [1], opto-couplers, etc. In such applications typically bandwidths below $100 \mathrm{MHz}$ are sufficient. This allows the use of PTs. However, it is important to analyze the photodetectors' noise, since the noise determines the sensitivity. Thus, it is advantageous and important to have a noise model of the PT for further circuit analysis.

\section{PhOTOTRANSISTORS, NoISE MODEL AND THEORY}

Four pnp pin PTs were used for the development and analysis of the noise model. Each PT was fabricated in a $0.6 \mu \mathrm{m}$ CMOS technology and has a thick, low doped p-epi collector layer, ensuring a thick space-charge region (SCR). This leads to an increased bandwidth and responsivity of the PT. Furthermore, the layout of the base and the emitter of each PT is unique, leading to different characteristics:

- $\mathrm{NW}_{50} \mathrm{E}_{\mathrm{ctr}}$ : half doped base, small center emitter.

- $\quad \mathrm{NW}_{50} \mathrm{E}_{\text {str }}$ : half doped base, stripped emitter.

- $\mathrm{NW}_{50} \mathrm{E}_{\text {full }}$ : half doped base, full emitter.

- $\mathrm{NW}_{\text {full }} \mathrm{E}_{\text {cor }}$ : full n-well base, small corner emitter.

Fig. 1 shows a $3 \mathrm{D}$ view of the $\mathrm{NW}_{50} \mathrm{E}_{\text {ctr }} \mathrm{PT}$. Further details on layout and characteristics of these PTs can be found in [2]. Fig. 2 depicts the developed noise model of a pnp pin PT in an open-base configuration, as used in the measurement setup. According to this model, the total output noise is composed of the base noise current and the collector noise current. Furthermore, since these currents are correlated a third additional noise term arises in the total output noise current formula [3]:

$$
\frac{\overline{l_{o_{P T}}^{2}}}{\Delta f}=\overline{\left(\beta \frac{l_{b}}{\sqrt{\Delta f}}+\frac{l_{c}}{\sqrt{\Delta f}}\right)^{2}}=\beta^{2} \frac{\overline{l_{b}^{2}}}{\Delta f}+\frac{\overline{l_{c}^{2}}}{\Delta f}+2 \Re\left\{C \sqrt{\beta^{2} \frac{\overline{l_{b}^{2}}}{\Delta f} \frac{\overline{l_{c}^{2}}}{\Delta f}}\right\} .
$$

Funding from the Austrian Science Fund (FWF) in the project P21373N22 is acknowledged.
As shown in (1) the base noise current is amplified by the squared current gain $\beta$ of the PT. $\beta$ is mainly dependent on the minority charge carrier density inside the base. This leads to a decrease of $\beta$ at higher collector currents (Kirk effect). The base noise current consists of a doubled shot noise part of the photocurrent, an $1 / f^{\gamma}$ noise part in the low-frequency range and the thermal noise part of the base resistance. The doubling of the shot noise part is caused by the open-base setup of the PT [4]. In this case the base-emitter current equals the photocurrent and their shot noise terms are quadratically summed, since they are uncorrelated. The collector noise current part is caused by the shot noise of the collector current at the output of the PT. In the third term of (1) $C$ describes the correlation between the first and the second term. It can take any value between -1 and +1 as stated in [3]. In the case of the presented open-base PTs $C$ has a value of 1 . This value is caused by the basecollector model resistor $r_{\mu}$, which acts like a source resistor with a high resistance in the $\mathrm{G} \Omega$ range. The high source resistor leads to a full correlation between the base noise current part and the collector noise current part [5]. Consequently, the high $\mathrm{G} \Omega$ value of $r_{\mu}$ prevents the thermal noise current contribution of the base resistance $r_{b}$. Thus, nearly the whole noise power of $r_{b}$ is negligible in the total output current noise $\overline{l_{o_{P T}}^{2}}$. Furthermore, $r_{\mu}, r_{\pi}$ and $r_{o}$ are model resistors and do not have any noise contribution.

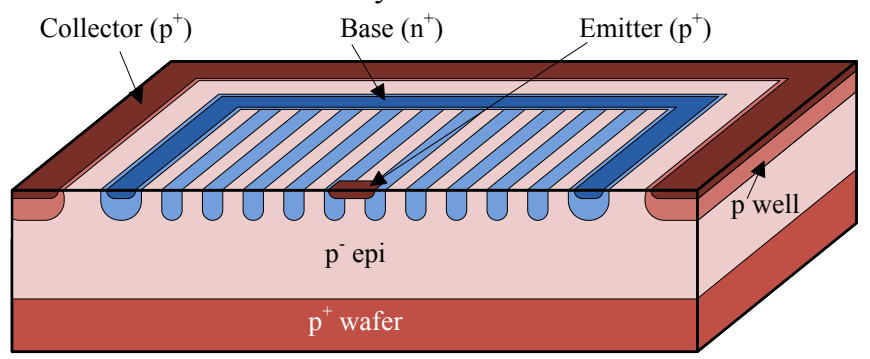

Figure 1. 3D depiction of the $\mathrm{NW}_{50} \mathrm{E}_{\text {ctr }}$ phototransistor.

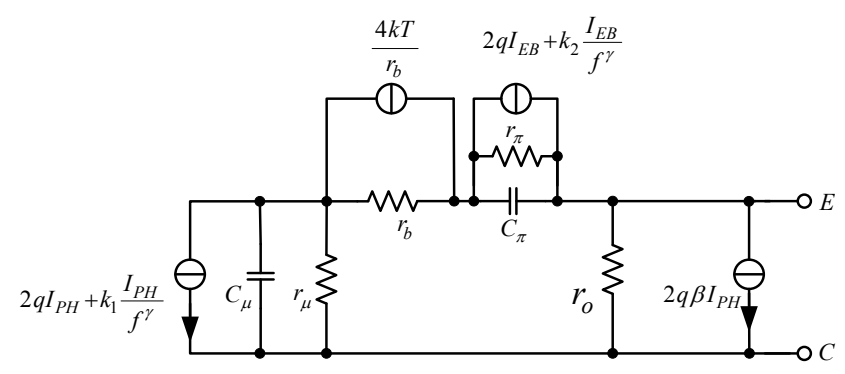

Figure 2. PNP phototransistor noise model. 


\section{MEASUREMENTS AND RESULTS}

The noise measurements were performed with the low noise operational amplifier (OPA) LT 1124 together with a $300 \mathrm{k} \Omega$ feedback resistor, a light bulb and a spectrum analyzer (SA). The OPA and the feedback resistor act together as a transimpedance amplifier and convert the output noise current of the PT into a noise voltage, which is captured by the SA. The measured output noise density was corrected by the noise contribution of the SA and the TIA.

Noise current density measurements of the $\mathrm{NW}_{50} \mathrm{E}_{\text {ctr }} \mathrm{PT}$ at different collector currents are presented in Fig. 3. The light power was adjusted to meet a defined collector current $(0.1 \mu \mathrm{A}$, $0.5 \mu \mathrm{A}, 1 \mu \mathrm{A}$ and $2 \mu \mathrm{A})$. The roll-off at higher frequencies is caused by the bandwidth limitation of the setup. Based on the model, the current gain $\beta$ of each PT was extracted after (1) from the average noise current density. To verify the correctness of the model, $\beta$ was measured by electrical Gummel measurements. Both $\beta$, the extracted one from the noise model and the measured one were compared and are depicted in Fig. 4. The comparison shows a good agreement of both. The deviation is below $9 \%$. This is a good value if considering the challenge of doing noise measurements and the typically limited precision. Furthermore, the total output white noise current density was modeled according to (1) using the $\beta$ from the Gummel measurements. A comparison of both noise densities is depicted in Fig. 5. The measured points represent the mean white noise current densities of the results presented in Fig. 3. All points except one are within a range of $12 \%$. Table I summarizes the presented values of the current gain $\beta$ and the output noise current of each PT at a collector current of $1 \mu \mathrm{A}$.

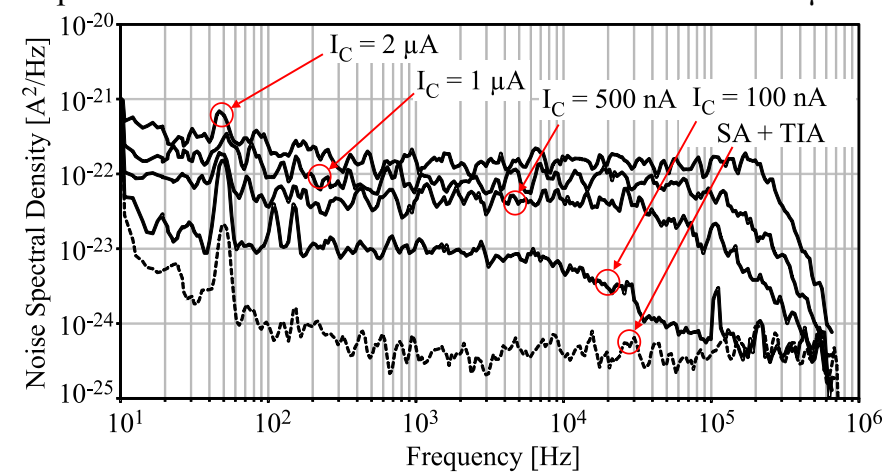

Figure 3. Output current noise spectral density of the $\mathrm{NW}_{50} \mathrm{E}_{\text {ctr }}$ phototransistor for different light intensities.

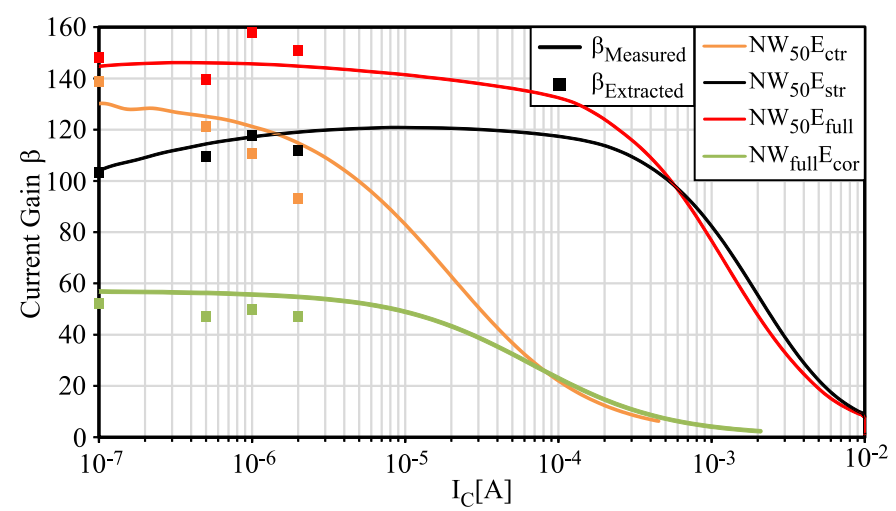

Figure 4. Measured and extracted current gain $\beta$.

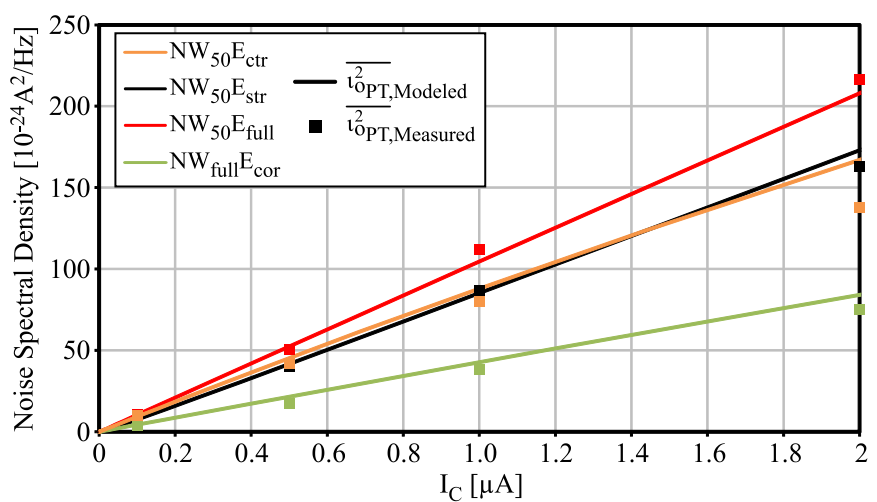

Figure 5. Measured and modeled output noise current at different $\mathrm{I}_{\mathrm{C}}$.

TABLE I. MEASURED AND MODELED VALUES FOR THE OUTPUT NOISE CURRENT IN $\left[10^{-24} \mathrm{~A}^{2} / \mathrm{W}\right]$ AND CURRENT GAIN $\beta$ AT $\mathrm{I}_{\mathrm{C}}=1 \mu \mathrm{A}$.

\begin{tabular}{lcccc}
\hline & $\overline{\mathbf{i}_{\text {OPT,Measured }}^{2}}$ & $\overline{\mathbf{i}_{\text {OPT,Modeled }}^{2}}$ & $\beta_{\text {Measured }}$ & $\beta_{\text {Extracted }}$ \\
\hline $\mathrm{NW}_{50} \mathrm{E}_{\text {ctr }}$ & 80.2 & 87.6 & 121 & 111 \\
$\mathrm{NW}_{50} \mathrm{E}_{\text {str }}$ & 86.5 & 86.1 & 117 & 118 \\
$\mathrm{NW}_{50} \mathrm{E}_{\text {full }}$ & 112.0 & 104.5 & 146 & 158 \\
$\mathrm{NW}_{\text {full }} \mathrm{E}_{\text {cor }}$ & 38.3 & 42.8 & 55 & 50 \\
\hline
\end{tabular}

\section{CONCLUSION}

A noise model for open-base configured phototransistors was developed and verified. The model and the verification are based on measurements done on four pnp pin phototransistors. Each device was processed in a $0.6 \mu \mathrm{m}$ CMOS process and has different characteristics. Phototransistor noise measurements were performed at four different collector currents. Furthermore, electrical Gummel measurements were performed to determine the current gain $\beta$ of each phototransistor. The model was verified by modeling the current gain $\beta$ and the output noise current from the noise current density and the Gummel measurements, respectively. Both, the modeled and the measured values were compared at the same collector currents. The comparison shows a difference of less than $12 \%$ between the modeled and the measured values of the current gain and the output noise current density.

\section{REFERENCES}

[1] J. Hurwitz, M.J. Panaghiston, K.M. Findlater, R.K. Henderson,T.E.R. Bailey, A.J. Holmes, B. Paisley, "A $35 \mathrm{~mm}$ film format CMOS image sensor for camera-back applications," IEEE International Solid-State Circuits Conference (ISSCC), vol.1, pp.48-443, 2002.

[2] P. Kostov, W. Gaberl, H. Zimmermann, "Visible and NIR integrated Phototransistors in CMOS Technology," Solid-State Electronics, vol. 65-66, pp. 211-218, 2011.

[3] D. Decoster, J. Harari, Optoelectronic Sensors, ISTE, John Wiley \& Sons, London, Hoboken, 2009.

[4] F.H. De La Moneda, E.R. Chenette, A. Van Der Ziel, "Noise in Phototransistors," IEEE Transactions on Electron Devices, vol.18, no.6, pp. 340-346, 1971.

[5] S.P.O. Bruce, L.K.J. Vandamme, A. Rydberg, "Measurement of LowFrequency Base and Collector Current Noise and Coherence in SiGe Heterojunction Bipolar Transistors Using Transimpedance Amplifiers," IEEE Transactions on Electron Devices, vol. 46, no. 5, pp. 993-1000, 1999. 